\title{
Statins: a revised appraisal for potential additional future treatment indications
}

\author{
Robert Weissert* \\ See related research by Vandebriel et al., http://arthritis-research.com/content/14/2/R90
}

\begin{abstract}
Beside statins' well established positive influence on atherosclerotic vascular disease caused by hypercholesterolemia through selective competitive inhibition of 3-hydroxy-3-methylglutaryl-coenzyme A (HMG-CoA) reductase, additional effects on the immune system have been described for them. These observations have raised great hopes for additional future treatment indications, including rheumatoid arthritis and multiple sclerosis. Ten years of searching for such novel treatment indications have not led to breakthroughs and future efforts must be seen with skepticism.
\end{abstract}

In the previous issue of Arthritis Research and Therapy Vandebriel and colleagues [1] report that the statins atorvastatin and pravastatin orally administered before disease induction in collagen-induced arthritis (CIA) in male DBA/lOlaHsd mice led to accelerated onset and higher disease incidence compared to controls. In addition, atorvastatin applied after induction of CIA resulted in earlier disease onset than administration before induction of CIA. Atorvastatin, but not pravastatin, administration also resulted in increased production of anti-collagen autoantibodies. In line with these novel findings in CIA, the same group recently demonstrated in a retrospective analysis of patients with rheumatoid arthritis (RA) that statins increase the risk for development of RA [2].

Statins are one of the most prescribed drugs in clinical medicine at present. Their main mechanism of action is selective, competitive inhibition of 3-hydroxy-3-methylglutaryl-coenzyme A (HMG-CoA) reductase, the rate-

*Correspondence: robert.weissert@klinik.uni-regensburg.de Department of Neurology, University of Regensburg, Universitaetsstrasse 84, 93053 Regensburg, Germany limiting enzyme that converts HMG-CoA to mevalonate, a precursor of sterols, including cholesterol.

Statins are indicated in patients with significantly increased risk for atherosclerotic vascular disease due to hypercholesterolemia for reduction of mortality from coronary heart disease, non-fatal myocardial infarction and stroke and reduction of coronary and non-coronary revascularization procedures. Furthermore, statins are indicated in patients with various forms of hyperlipidemia. While statins have a clear and well established positive effect in the disease conditions named above, emerging data for additional indications is rather weak and side effects should be taken into consideration.

In addition to the inhibition of HMG-CoA reductase, a multitude of additional drug-related and off-target effects with possible therapeutic value have been described. Importantly, it has also been demonstrated that statins have immunomodulatory properties. Most prominently, atorvastatin application in experimental autoimmune encephalomyelitis, the animal model of multiple sclerosis, leads to reduced disease severity due to effects on antigen presentation and T-cell activation and phenotype [3]. Unexpectedly, clinical studies in multiple sclerosis patients could not confirm the beneficial effects of statins observed in experimental autoimmune encephalomyelitis [4]. Statins that pass the blood-brain barrier should also be used with caution for other reasons, since some experimental data indicate that statins inhibit remyelination [5].

Statins have been evaluated as a treatment of Alzheimer's disease. There are positive trends, but the data are conflicting and further studies in patients with mild Alzheimer's disease will possibly clarify the assumed therapeutic value [6].

The findings of Vandebriel and colleagues are in contrast to previously published reports in which atorvastatin, pravastatin, simvastatin and rosuvastatin given by various routes after CIA induction in mice have been reported to reduce disease or to have no effect (summarized in [1]). The reasons for the discrepancy could be differences in disease induction, route of dosing and dose, used substrains of mice and the animal housing 
environment. On the other hand, there is no previously published study in which statins were given before disease induction of CIA.

So far statins have also been considered of potential future therapeutic value in RA. In RA there are data indicating that atorvastatin as an add on therapy to disease-modifying antirheumatic drugs (DMARDs) has beneficial effects in patients with RA: the randomized double blind placebo-controlled Atorvastatin in Rheumatoid Arthritis (TARA) study demonstrated some modest effects on acute phase variables and swollen joints, while other parameters remained unaffected [7]. Another study equally demonstrated beneficial effects in patients with RA treated with atorvastatin and reported an increased number of regulatory $\mathrm{T}$ cells as a possible mechanism of action [8]. Others reported effects on inflammatory parameters and Th1/Th2 balance leading to beneficial treatment effects of simvastatin in RA [9].

Recently, the US Food and Drug Administration (FDA) changed its safety label for statins and indicated that liver injury associated with statin use is rare but can occur. They also acknowledged that statin use can be associated with memory loss, forgetfulness and confusion. In addition, a small, increased risk of raised blood sugar levels and the development of type 2 diabetes have been reported. Moreover, there is a risk for muscle damage.

Vandebriel and colleagues also demonstrate an influence on collagen type II autoantibody titers after treatment with atorvastatin [1]. Since this was only the case for atorvastatin and not pravastatin, the data could indicate varying effects on the immune response of different types of statins. Possibly the B-cell arm of autoimmune diseases could be affected by statin administration, leading to increased autoantibody titers. In line with the speculation that the B-cell arm of the immune response is affected by statin administration is the increased incidence of lupus-like syndromes in patients receiving statins [10].

In summary, the work by Vandebriel and colleagues [1] suggests that broad use of statins could lead to increased incidence of autoimmune disease, especially RA. Further work will be necessary to demonstrate the cell biological and immunological mechanisms explaining these findings. Future studies in rodents and humans should better delineate the differences in the properties of individual statins with regard to their modes of action. The hypothesis that statins will have additional future indications for treatment of autoimmune diseases like RA or multiple sclerosis is more unlikely. Presently, there are no convincing data for the use of statins in these indications outside of controlled clinical trials. Exceptions remain co-morbidities with cardiovascular disease, stroke and hyperlipidemia.

\section{Abbreviations \\ CIA, collagen-induced arthritis; HMG-CoA, 3-hydroxy-3-methylglutaryl- coenzyme A; RA, rheumatoid arthritis.}

\section{Competing interests}

The author declares that they have no competing interests (financial and non-financial).

Published: 29 June 2012

\section{References}

1. Vandebriel RJ, de Jong HJ, Gremmer ER, Klungel OH, Cohen Tervaert JW, Slob W, van der Laan JW, van Loveren H: Statins accelerate the onset of collagen type Il-induced arthritis in mice. Arthritis Res Ther 2012, 14:R90.

2. de Jong HJ, Klungel OH, van Dijk L, Vandebriel RJ, Leufkens $H G$, van der Laan $J$, Tervaert JW, van Loveren H: Use of statins is associated with an increased risk of rheumatoid arthritis. Ann Rheum Dis 2012, 71:648-654.

3. Youssef S, Stüve O, Patarroyo JC, Ruiz PJ, Radosevich JL, Hur EM, Bravo M, Mitchell DJ, Sobel RA, Steinman L, Zamvil SS: The HMG-CoA reductase inhibitor, atorvastatin, promotes a Th2 bias and reverses paralysis in central nervous system autoimmune disease. Nature 2002, 420:78-84.

4. Sorensen PS, Lycke J, Erälinna JP, Edland A, Wu X, Frederiksen JL, Oturai A, Malmeström C, Stenager E, Sellebjerg F, Sondergaard HB; SIMCOMBIN study investigators: Simvastatin as add-on therapy to interferon beta-1a for relapsing-remitting multiple sclerosis (SIMCOMBIN study): a placebocontrolled randomised phase 4 trial. Lancet Neurol 2011, 10:691-701.

5. Miron VE, Zehntner SP, Kuhlmann T, Ludwin SK, Owens T, Kennedy TE, Bedell BJ, Antel JP: Statin therapy inhibits remyelination in the central nervous system. Am J Pathol 2009, 174:1880-1890

6. Shepardson NE, Shankar GM, Selkoe DJ: Cholesterol level and statin use in Alzheimer disease: II. Review of human trials and recommendations. Arch Neurol 2012, 68:1385-1392.

7. McCarey DW, McInnes IB, Madhok R, Hampson R, Scherbakov O, Ford I, Capell HA, Sattar N: Trial of Atorvastatin in Rheumatoid Arthritis (TARA): doubleblind, randomised placebo-controlled trial. Lancet 2004, 363:2015-2021.

8. Tang TT, Song Y, Ding YJ, Liao YH, Yu X, Du R, Xiao H, Yuan J, Zhou ZH, Liao MY, Yao $R$, Jevallee $H$, Shi GP, Cheng X: Atorvastatin upregulates regulatory $T$ cells and reduces clinical disease activity in patients with rheumatoid arthritis. J Lipid Res 2011, 52:1023-1032.

9. Kanda H, Yokota K, Kohno C, Sawada T, Sato K, Yamaguchi M, Komagata Y, Shimada K, Yamamoto K, Mimura T: Effects of low-dosage simvastatin on rheumatoid arthritis through reduction of Th1/Th2 and CD4/CD8 ratios. Mod Rheumatol 2007, 17:364-368.

10. Moulis G, Bene J, Sommet A, Sailler L, Lapeyre-Mestre M, Montastruc JL, Regional Pharmacovigilance Centers FA: Statin-induced lupus: a case/noncase study in a nationwide pharmacovigilance database. Lupus 2012, 21:885-889.

\section{doi:10.1186/ar3880}

Cite this article as: Weissert R: Statins: a revised appraisal for potential additional future treatment indications. Arthritis Research \& Therapy 2012, 14:121. 